\title{
Estimation-Based Noise-Robust Sensing
}

\author{
Andreas Polydoros* and Ioannis Dagres \\ Institute of Accelerating Systems \& Applications \\ National Kapodistrian University of Athens \\ Athens, Greece \\ \{polydoros, jdagres\}@phys.uoa.gr
}

\begin{abstract}
It is well known that noise-modeling uncertainties give rise to a fundamental limit on the sensitivity of energy-based detectors (or related moment-based detectors), also known as the "SNR wall". Similar "walls" appear also when localizing unknown sources for various applications (including cognitive radio), since localization techniques are based on tempo-spatial signal features. Low SNR regimes plus vague knowledge of the characteristics of the signal under detection in such applications motivate the exploration of techniques that are robust to such parameter uncertainties and therefore immune to such limits. A simple conceptual framework is proposed herein that naturally generates techniques with such immunity advantages for detecting the presence of an unknown source in broadband noise At the cost of longer observation time, they allow SNR-wall-free detection. They also lend themselves to precise analysis and parametric optimization.
\end{abstract}

\section{Keywords-detection;localization;features;}

\section{INTRODUCTION}

Source sensing, meant as classic binary hypothesis testing (presence detection) of a source in a given spectral band, an important function in cognitive radio and other applications, must typically be performed in the absence of detailed knowledge of at least some aspect of the system and signal model. This lack of knowledge may range from simply not knowing some of the channel characteristics, such as the channel complex gain in a flat-fading channel (but knowing precisely either the signal shape or some parts of the signal, e.g., its pilots or preambles), all the way to knowing nothing about the sought signal or the intervening channel. The latter case is the easiest to handle, meaning that then one can only resort to non-coherent, moment-based processing (energy detection, autocorrelation, spectral correlation/cyclo-stationary processing, etc.). This latter class of techniques is popular with the sensing community because it demands the least from the algorithm designer, although it is still subject to optimization of its various parameters (for instance, which correlation or cyclic-frequency terms to incorporate in the detection statistic and with what weight). Still, it has the appeal of simplicity, invoking the least set of assumptions, and it may work well for high enough SNR. In addition, energy (quadratic) detection is indeed mathematically optimal under very specific modeling assumptions (Gaussian signal in Gaussian noise). If the signal is not patently Gaussian but unknown in its features, it still makes sense as an "agnostic" solution [1]. The difficulty with energy detection is that it is brittle when the noise level is not precisely known, because then the false-alarm-based threshold cannot be set with accuracy. This leads to the known "SNR wall" phenomenon [2], [3]. Ways to somewhat bypass this sensitivity to the relative ignorance of the noise level have been explored in depth and tend to center on autocorrelation or spectral-correlation techniques that omit the zero-lag term, which includes the mean value of the noise level (although there is still dependence on the noise level to set the threshold, just less) [4].

On the other extreme, the signal may be known in shape, including the values of its data symbols for some duration (for instance, pilot symbols). This can be exploited in a matchedfilter or signal-correlation operation, akin to what is done in coherent receivers for digital communication operation. The result will be a coherent accumulation of voltage during this correlation interval and a concomitant increase in collected energy versus the purely sample-by-sample non-coherent processing of the energy detector (which suffers the so-called "non-coherent combining loss"). Therefore, matched filtering which exploits such knowledge will result in an SNR shift for performance (an SNR benefit). We note, however, a fundamental difference between coherent filtering in coherent comm. receivers and pseudo-coherent processing in sensing receivers: the latter cannot exploit the exact channel knowledge (gain and phase) enjoyed by a coherent comm. receiver due to the carrier synchronization/equalization preprocessing that the comm. receiver enjoys. As a result, there will be a non-coherent (complex-norm) operation at the end of the sensing receiver processing, and therefore, in that sense, all sensing receivers are non-coherent in nature; there is no such thing as a fully coherent sensing receiver. The only difference between classic non-coherent processing and matched-filter processing is whether the squaring operation takes place sample by sample or at the end of the observation interval.

Here, a new framework for Constant-False-Alarm-Rate (CFAR), feature-based detection is proposed. We focus on (but are conceptually not limited to) spatial signal features that arise due to the reception of the signal at different points in space. These features allow detection (first, and that is the novelty) as well as subsequent localization of unknown sources based on an estimation pre-processor. The proposed family of statistical tests is based on the appropriate processing of the received samples in order to create successive and statistically independent estimates of signal features that have meaning if and only if a signal is present.

This work has been supported by the FARAMIR project (ICT-248351), funded by the European Commission.

*Also a distinguished adjunct professor of King Abdulaziz University, Jeddah, Saudi Arabia. 
By first quantizing the feature range and subsequently estimating the most probable value for the feature under consideration, a histogram is created which signifies the relative probabilities for the different (discrete) sub-regions on the value of the feature. Based on an analytical description of this histogram, a test is created on the existence or not of the signal, as well as the value of the feature (as an immediate fridge benefit). The whole procedure takes therefore two steps: the first where successive estimates of a feature are made as if the signal exists and a second where these estimates are employed in order to decide on the binary hypothesis of the signal existence, as well as the feature value. A large number of tests that assume accurate knowledge of the noise level have been proposed [1][5]. These tests are expected to have better performance than the one proposed but suffer from SNR walls. Tests that are CFAR without the knowledge of the noise level have also been proposed, [4],[6], that use a selfnormalizing approach but are difficult to analyze and thus optimize. Here, by locating the position (in feature space) of the maximum of the histogram as the appropriate statistic for the final binary test, a decision is made on the existence of a signal, as well as on the value of the feature should the decision be positive; otherwise, this value is meaningless. It is also shown here that the analysis of the performance of this scheme is straightforward, thus allowing for subsequent optimization of the test.

This paper is organized as follows: In section II the histogram test is described, which a fundamental part of the proposed approach. Besides the description, an approximate analysis is provided along with simulation results for assessing its accuracy. Two application examples in Section III demonstrate the breadth of the concept, one for cyclic-prefixbased (CP) detection plus one for time difference of arrival (TDOA) -based detection and localization. The details for proper data processing, together with an analytic performance description are provided. Simulation results and final conclusions are given at section IV and V, respectively.

\section{THE HistOgRAM TEST}

In this section we will describe a simple, non-linear test that will be used as the cornerstone for the proposed approach. Within this framework, different localization as well as feature estimation techniques already available in the literature can be employed. A test description is provided at a fairly abstract level, with specific instantiations described in the following Sections for clarity.

\section{A. Analysis}

We address the following binary hypothesis problem: under $\mathbf{H}_{\mathbf{0}}$ (no signal present) we observe the outcome of a purely random choice between $N_{L}$ events, denoted by $h_{i}\left(i=1, \ldots, N_{L}\right)$, all with equal probability. Under $\mathbf{H}_{\mathbf{1}}$ (signal present) one specific event, denoted by $h_{c}$, has a larger probability of occurrence than the rest. This is the simplest scenario that we analyse here; extensions to multiple events of higher probability are under investigation. Let $y_{k}$ be the $k$-th observation. Then, $y_{k}=h_{i}$ where, for $\mathbf{H}_{\mathbf{0}}$,

$$
p\left(h_{i}\right)=\frac{1}{N_{L}}
$$

whereas for $\mathbf{H}_{1}$,

$$
p\left(h_{c}\right)=p, p\left(h_{i}\right)=\frac{1-p}{N_{L}-1}<p \text { for } h_{i} \neq h_{c}
$$

Here $p\left(h_{i}\right)$ is the probability of observing the event $h_{i}$. After $N_{b}$ observations we wish to decide on the prevailing hypothesis for a given target probability of false alarm $P_{f a}$. At the same time, an estimate of the event with the highest probability of occurrence (under $\mathrm{H}_{1}$ ) is produced.

The following test is proposed for the just-described joint detection /estimation problem: Define

$$
T=\max (\operatorname{hist}(\mathbf{y})) \lessgtr \lambda
$$

the test for the detection task, and $\hat{c}=\operatorname{index}(T)$ for the estimation, where $\mathbf{y}=\left[y_{1}, y_{2}, \ldots, y_{N_{b}}\right]$ is the total vector of observations, hist( $\cdot)$ is the histogram operator, namely a counter for the number of occurrences for each of the $N_{L}$ events, $\max (\cdot)$ is an operator that provides the value of the largest number of the input vector and index $(\cdot)$ is its index. Other tests could also be devised with possibly better performance, but the currently proposed test is simple and performs almost optimally in the parameter regions of interest. For $\mathbf{H}_{\mathbf{0}}$, hist(y) is a length- $N_{L}$ vector of random variables (rv), $\widehat{h}_{i} \in\left[0,1, . ., N_{b}\right]$, each following a binomial distribution with probability density function (pdf)

$$
p\left(\widehat{h}_{i}=x\right)=\left(\begin{array}{c}
N_{b} \\
x
\end{array}\right) p\left(h_{i}\right)^{x}\left(1-p\left(h_{i}\right)\right)^{N_{b}-x},
$$

with $x=0,1, \ldots, N_{b}$ and its cumulative distribution function (cdf)

$$
F_{i}(x)=P\left(\widehat{h}_{i} \leq x\right)=\sum_{r=0}^{x}\left(\begin{array}{c}
N_{b} \\
r
\end{array}\right) p\left(h_{i}\right)^{r}\left(1-p\left(h_{i}\right)\right)^{N_{b}-r},
$$

To analyze the performance of the proposed test, the cdf of the maximum of these $N_{L}$ rv's is required. A simple approximation can be provided by ignoring their correlation. The cdf of the maximum is given by

$$
F_{N_{L}: N_{L}}(x)=F_{i}(x)^{N_{L}}
$$


Using this approximation, the $\mathrm{P}_{\mathrm{f}}$ for a threshold value $\lambda$ is

$$
P_{f}(\lambda)=1-F(\lambda)^{N_{L}}
$$

Using the same rationale for the $\mathrm{H} 1$ case, the probability of detection $\left(P_{d}\right)$ is approximated by

$$
P_{d}(\lambda)=1-F_{c}(\lambda) F_{i}(\lambda)^{N_{L}-1}
$$

\section{B. Accuracy of approximations}

We now examine the accuracy of the above approximations for this type of test, since it is the most critical part for guaranteeing the level of false alarm while optimizing the probability of detection. The discrete distributions that describe both hypotheses are generated by $N_{L}$ uniform and continuous rv's, where the maximum is chosen at each sample test. In order to produce the required statistics for the $\mathrm{H}_{1}$ case, $\left(N_{L}-1\right)$ rv's are generated with support in $[0,1]$ plus one in $[0+s, 1+s]$, where $s$ is chosen so that the probability of indeed being the maximum in the set equals $p$. This is done by solving numerically the following equation for $p: p=\left(1-s^{N_{L}}\right) / N_{L}+s$.

By producing the $N_{L}$ rv's for each hypothesis and choosing the index $i$ of the maximum, we can produce the $h_{i}$ 's that fit the above scenario for any arbitrary values of $p, N_{L}, N_{b}$.

We now demonstrate the approximation of the analysis and the performance of the test for some indicative values of the aforementioned parameters. In Fig. 1 we demonstrate the accuracy of (8) for $N_{L}=100$ and $N_{b}=10,100,1000$.

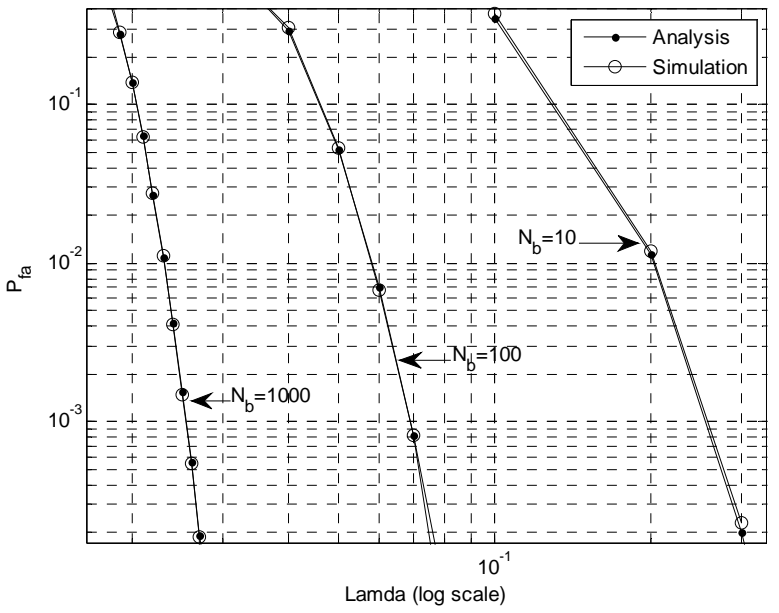

Figure 1. Probability of false alarm vs lamda

In Fig. 2, we demonstrate the accuracy of (9) for $N_{L}=10$, $N_{b}=100$ and $p_{c}=0.2,0.4$ and 0.6. We conclude that the proposed analysis follows the simulation results with very high accuracy for the discussed cases; all other simulations performed extensively have the same behavior (not shown here). All basic tools are now in place to achieve a target ROC, with the classic definition of ROC $=\left(P_{f}, P_{d}\right)$. We now proceed to show specific instantiations of the theory for detection (only) as well as joint detection/localization in examples of common interest.

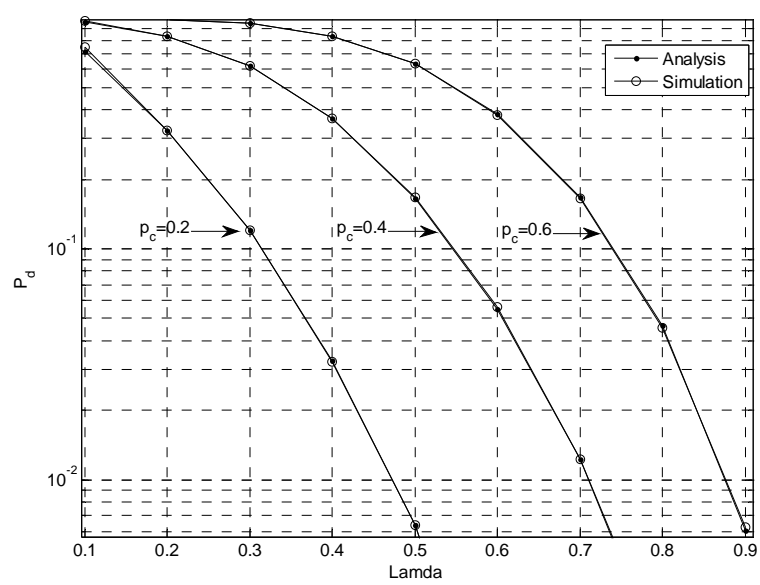

Figure 2. Probability of detection vs lamda

\section{APPLICATION EXAMPLES}

The first example involves the detection of an OFDM source based on its cyclic prefix (CP). The second involves the joint detection/localization of a source based on time TDOA estimation. Both examples can be treated in a unified manner under the proposed framework. The only difference (besides parameterization constraints) is what feature is chosen to represent the test. In the first example it is the symbol synchronization time, while in the second it is the TDOA between two different points in space. Both exhibit a periodicity: due to the symbol rate in the first case and due to the spatial coherence bandwidth of a source in the second.

\section{A. Cyclic prefix based OFDM detection}

Let $N_{c p}$ be the cyclic prefix length, $N_{\text {FFT }}$ the FFT length, and $N_{S \text {-total }}$ the total number of OFDM symbols received. We assume the signal bandwidth and the above parameters known. The proposed processing is depicted in Fig. 3. Since the starting time of a symbol is unknown, the ambiguity range is $N_{c p}+N_{\text {sym }}$, equal to the previously defined $N_{L}$. For each of the $N_{L}$ possible events, the following two vectors are defined: $\mathbf{r}_{1, i}=\left[\ldots, y\left(i+k\left(N_{c p}+N_{s y m}\right)\right), \ldots\right]$ and $\mathbf{r}_{2, i}=\mathbf{r}_{1, i+N_{c p}}$, with $k \in\left(0, . . N_{S-t o t a l}\right) ; i \in\left(1, . . N_{c p}+N_{\text {sym }}\right)$. Classic estimation of the symbol starting time involves the inner product of $\mathbf{r}_{1, i}$ with $\mathbf{r}_{2, i}$ for all possible candidate values of $i$. In low SNR, it has been shown [8] that it suffices to use the real part of the inner product, i.e.

$$
\hat{t}=\arg \left(\max \left(\operatorname{real}\left(\mathbf{r}_{1, i}^{H} \mathbf{r}_{2, i}\right)\right)\right)
$$




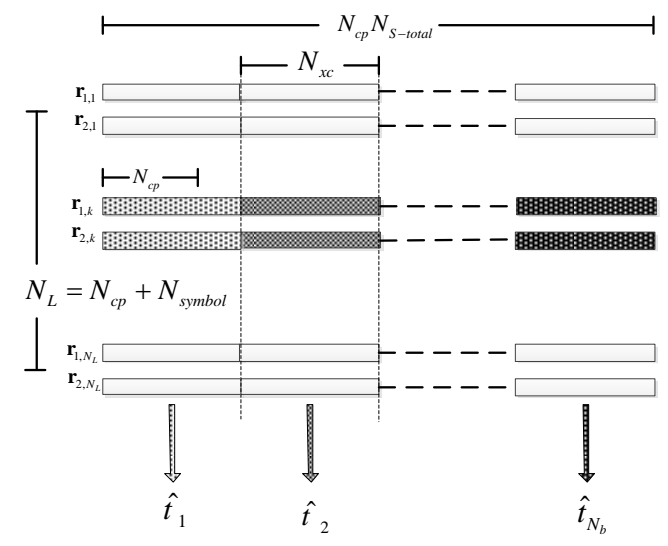

Figure 3. Proposed processing for cp-based detection example

As shown in Fig. 3, $N_{x c}$ is the number of samples employed here for each estimate $\left(1 \leq N_{x c} \leq N_{s}\right)$. The constraint imposed by the finite number of samples is described by the following inequality $N_{b} N_{x c} \leq N_{s}$. We can now compute, for any set of desirable parameters, the threshold that guarantees the desired $P_{f a}$. In addition, knowledge of the SNR level, with any level of accurancy, enables parameter optimization for a target ROC.

\section{B. TDOA based source detection/localization}

Here the proposed framework is adopted as a test for detecting a source via TDOA estimation. The envisioned scenario assumes multiple pairs of sensors doing an identical test, then properly combining the individual TDOA estimates for a final statistic. The focus here is on the intermediate step of individual TDOA estimations, not the final geometric transformation of these multiple estimates to localization.

Let $r_{1}, r_{2}$ be the two received sequences by two spatially distinct sensors, both of which either observe noise only or signal plus noise. The signal, if present, is modeled as a white process (extension to colored signals is straightforward). Then, employing classic cross-correlation-based estimation as shown in (10), the lag corresponding to the maximum value is the candidate TDOA estimate.

This is conceptually equivalent to an autocorrelationfeature detection test, since it is still the same (noiseless) signal that is received at both sensors under $\mathrm{H}_{1}$. Instead of trying to solve for the optimal detector under some (partial) knowledge of the noise statistics, the proposed framework is again engaged, leading to a true CFAR test because the $P_{f a}$ is fully independent of the noise level. Fig. 4 demonstrates how statistically independent TDOA estimates can be generated. Clearly, at least two estimates are needed for non-trivial tests. Let $N_{L}$ be the range of values of TDOA, $N_{s}$ be the total number of samples, $N_{b}$ be the number of TDOA estimates, and $N_{x c}$ be the number of samples used for producing each estimate. These parameters are constrained by the inequality $N_{b} N_{x c}+N_{L} \leq N_{s}$.

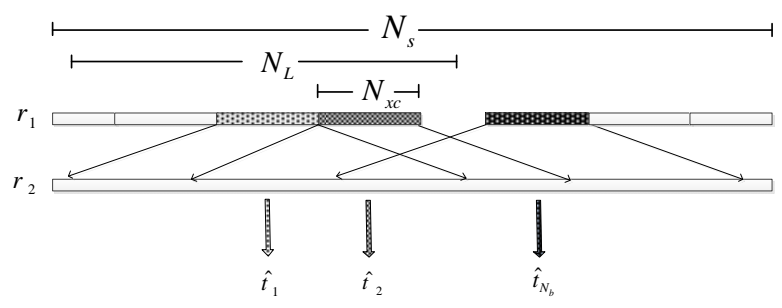

Figure 4. Proposed processing for TDOA-based detection example

The previous description of the histogram-based test indicates that the probability of successful detection $P_{d}$ can be maximized for a given $P_{f a}$ level, under parametric choices that are subject to some constraints. From an estimation-only point of view, TDOA accuracy increases with $N_{x c}$. But since at least two estimation samples are needed for a non-trivial test, a certain degradation of TDOA estimation accuracy is expected. It will soon be evident that a trade-off exists between the two tasks of detection (signal detectability) and final estimation (localization accuracy). We note, however, that such a tradeoff is eventually artificial and rather created by system-level constraints such as processing complexity, data-exchange bandwidth, etc. It is not a fundamental law, in other words, since estimation/localization can be re-performed after successful detection by optimally employing the whole data set. In that sense, parameter estimation is understood here chiefly as a detection pre-processor, leading to the concept of estimation-based detection.

\section{PERFormance Assessment}

To analyze performance, the description of the test statistics should be derived. The distribution of the sum of products of normal r.v's resulting from the cross-correlation operation has been described in [8], where a Gaussian approximation is used based on the central-limit theorem. In [8], a CP-based detector is described, leading to an analysis that is applicable to the present one. As mentioned before, at low SNR, only the real part of the cross-correlation need to be employed with very small degradation in performance. The relevant test statistics can be approximated as $N\left(0, \sigma^{2}\right), \forall i$ for the H0 case, while for the H1, $N\left(\mu, \sigma^{2}\right)$ for $i=c$ and $N\left(0, \sigma^{2}\right)$ for $i \neq c$, where $\sigma^{2} \approx \sigma_{n}^{2} / 2 N_{L}, \mu=\sigma_{s}^{2}$, with $\sigma_{n}^{2}$ the variance of the complex additive Gaussian noise and $\sigma_{s}^{2}$ the variance (power) of the Gaussian-modeled signal. For $\mathrm{H}_{0}$, since all $h_{i}$ 's possess the same distribution, the probability that any one of them corresponds to the maximum has been derived in Section II.. Thus, $\mathrm{P}_{\mathrm{fa}}$ is described by (7). For $\mathrm{H}_{1}$, the probability of choosing the right lag, $p\left(h_{c}\right)$, must be derived in order to be able to compute and optimize $\mathrm{P}_{\mathrm{d}}$. This probability is, in fact, the familiar probability of correct decision of M-ary orthogonal signals in additive Gaussian noise, given by

$$
\mathrm{p}_{c}\left(\mu, \sigma^{2}, N_{L}\right)=\frac{1}{2 \pi \sigma^{2}} \int_{-\infty}^{\infty}\left(\int_{-\infty}^{a} \mathrm{e}^{-\frac{z^{2}}{2 \sigma^{2}}} \mathrm{~d} z\right)^{N_{L}} \mathrm{e}^{-\frac{1}{2 \sigma^{2}}(a-\mu)^{2}} d a
$$




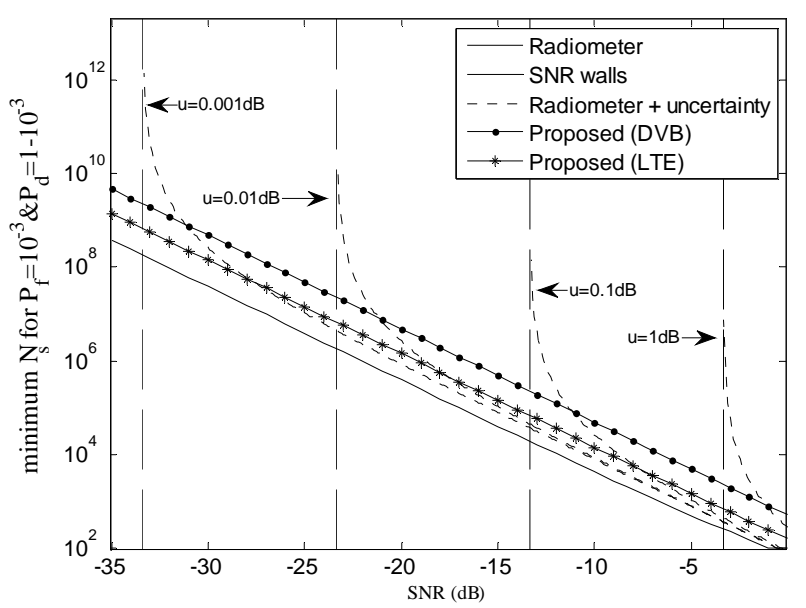

Figure 5. Sample size versus SNR

Many approximations are known for computing the above integral [9]. Unfortunately, they all target bit error probabilities which must be very small to be useful, thus not suited for the present detection problem. Numerical algorithms for approximating (11) for the parameter ranges of interest have been employed in this paper.

An identical line of performance analysis applies to both examples. Here, due to space limitations, we analyze only the first. We employ typical OFDM values from the DVB standard, such as $N_{c p}=1024, N_{F F T}=8192$ or from the LTE standard, such as $N_{c p}=36, N_{F F T}=512$. Fig. 5 plots the required sample size as a function of SNR for achieving a given ROC. The purpose is to access the new test performance as compared to the radiometer when the latter operates either under ideal conditions (perfectly known noise level) or nonideal ones (uncertainty in the noise level), thus demonstrating the immunity of the proposed class of tests to noise uncertainty. We conclude that the new tests are comparatively inefficient under ideal conditions (an SNR loss versus the ideal radiometer under no noise-level uncertainty). But when a u-parameterized uncertainty on the noise level exists, the radiometric detectors are 'tuned' to the worst-case noise level for guaranteeing the $P_{f a}$ level (see [3] for details). Then, the proposed scheme becomes superior and exhibits no wall; the only effect is an SNR loss, proportional to $u$ (in $\mathrm{dB}$ ). The worst case plotted is for $\mathrm{u}=1 \mathrm{~dB}$, causing a radiometric SNR wall at about $-3 \mathrm{~dB}$, while the proposed test improves continuously with SNR.

Since non-radiometric detectors in general are affected by limited processing time due to channel coherence, Figure 6 demonstrates the performance degradation of the proposed scheme based on a maximum interval for coherent processing. It can be seen that a maximum value for $N_{x c}$ results in a slope change at a certain SNR point. Performance degrades, but still no SNR wall appears.

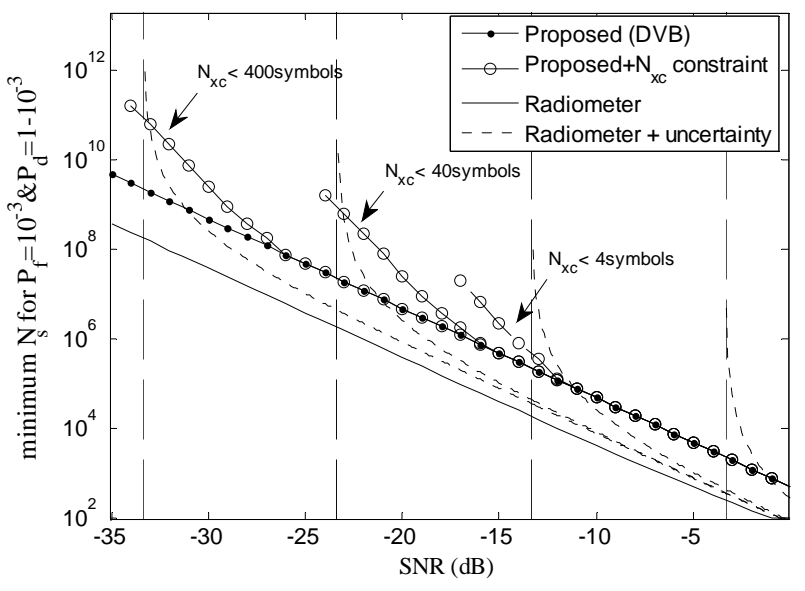

Figure 6. Sample size versus SNR

\section{CONCLUSIONS}

A new framework for source detection and approximate localization has been proposed, based on a simple idea: first estimate appropriately-chosen parameters and then use the results to perform detection (a reversal of usual thinking). The test uses successive and independent estimates of signal features to decide upon the presence or absence of a signal. A highly accurate approximate analysis of the proposed test has been provided, enabling performance characterization and optimization. Two application examples have been provided that demonstrate the use of the concept. Extensions of the test for more challenging scenarios of multiple sources as well as realistic system models are currently under investigation.

\section{REFERENCES}

[1] H. Urkowitz, "Energy Detection of Unknown Deterministic Signals," Proceedings of the IEEE, April 1967, pp. 523-531.

[2] R. Tandra, and A.Sahai, "SNR Walls for Feature Detectors " IEEE DySPAN 2007 Issue Date: 17-20 April 2007 On page(s): 559 - 570

[3] R. Tandra and A. Sahai, "SNR walls for signal detection," IEEE Journal on Selected Topics in Signal Processing, pp. 4-17, Feb 2008.

[4] T. Ikuma and M.Naraghi-Pour, "A Comparison of Three Classes of Spectrum Sensing Techniques", IEEE GLOBECOM 2008.

[5] F. Digham, M-S. Alouini, M.K Simon, M. K. "On the Energy Detection of Unknown Signals Over Fading Channels", IEEE Transactions on Communications, Jan. 2007, Vol. 55 Issue:1, pp. 21 - 24

[6] M. Naraghi-Pour and T. Ikuma, "Autocorrelation-Based Spectrum Sensing for Cognitive Radios", IEEE Transactions on Vehicular Technology,Issue Date: Feb. 2010.

[7] Y. Zeng and Y. Liang, "Covariance based signal detections for cognitive radio,” in 2nd IEEE DySPAN, Dublin, Ireland, 2007, pp. 202-207.

[8] C. Sachin, L. Jarmo, K. Visa, "Collaborative Autocorrelation-Based Spectrum Sensing of OFDM signals in Cognitive Radios", 42nd Annual Conference on Information Sciences and Systems, 2008. CISS 2008.

[9] K. Karil, D. Chawla, V. Sarwate, "Upper Bounds on the Probability of Error for M-ary Orthogonal Signaling in white Gaussian noise" IEEE Trans. on INF.Theory, vol 36, no. 30, May 1990. 